\title{
ERCP in patients with COVID-19 infection - is a single-use duodenoscope the safer option?
}

We read with interest the article by Zhang et al. regarding suggestions for infection prevention and control in digestive endoscopy during the COVID-19 pandemic in Wuhan, China [1]. All upper gastrointestinal endoscopy procedures are aerosol generating, hence there is risk of spreading infection [2]. Endoscopic retrograde cholangiopancreatography (ERCP) carries the additional risk of duodenoscope-related infection [3,4]. Recently, the US Food and Drug Administration approved the use of disposable duodenoscopes. We report performing an ERCP in a patient with cholangitis who was under investigation for COVID19 using a single-use duodenoscope. The patient was a 90-year-old woman with a past history of congestive heart failure and dementia who presented with fever and abdominal pain. She was tested for COVID-19. Physical examination, laboratory data, and imaging were suggestive of acute cholangitis and septic shock. Therefore, we proceeded to ERCP and, given that the patient was under investigation for COVID-19, she was presumed to be positive as per our institutional guidelines. A single-use duodenoscope (EXALT Model D; Boston Scientific Corporation, Marlborough, Massachusetts, USA) was used for the procedure. The common bile duct was cannulated and a cholangiogram revealed choledocholithiasis. A biliary sphincterotomy was deferred because of clopidogrel use and a plastic biliary stent was placed. The procedure duration was 15 minutes. Upon completion, the endoscope was disconnected from the processor and sealed in a bag, and the room was disinfected.
Single-use duodenoscopes could be beneficial in COVID-19 patients requiring ERCP. The use of a disposable duodenoscope eliminates the low risk of patientto-patient transmission related to inadequate disinfection. There is also a risk of aerosolization during disinfection and therefore of exposure risk to personnel performing endoscope cleaning, both in the procedure room and in the endoscope cleaning room. Furthermore, the theoretical risk of aerosolization during endoscope disinfection could contaminate the cleaning room necessitating its closure for 30 minutes after the disinfection process is completed.

Using a single-use duodenoscope for ERCP in patients with confirmed or suspected COVID-19 may be a reasonable strategy to reduce the risk of infection among patients and healthcare personnel. However, more data are needed to see if SARS-Cov-2 specifically is difficult to remove from duodenoscopes, and considerations of cost-effectiveness and environmental impact of single-use devices will need to be taken into account.

\section{Competing interests}

Tyler Berzin is a consultant for Boston Scientific and Medtronic. Douglas Pleskow is a consultant for Boston Scientific, Medtronic, Olympus, and Fuji. The remaining authors declare that they have no conflict of interest.

The authors

Mohammad Bilal, Tyler M. Berzin, Jonah Cohen, Mandeep S. Sawhney, Douglas K. Pleskow

Center for Advanced Endoscopy, Division of Gastroenterology, Beth Israel Deaconess Medical Center, Harvard Medical School, Boston, Massachusetts, USA

\section{Corresponding author}

Douglas K. Pleskow, MD

Harvard Medical School, Center for

Advanced Endoscopy, Beth Israel Deaconess

Medical Center, 330 Brookline Avenue,

Boston, MA 02215, USA

Fax: +1-617-632-9199

dpleskow@bidmc.harvard.edu

\section{References}

[1] Zhang $Y$, Zhang $X$, Liu L et al. Suggestions for infection prevention and control in digestive endoscopy during current 2019-nCoV pneumonia outbreak in Wuhan, Hubei province, China. Endoscopy 2020; 52: 312-314

[2] Repici A, Maselli R, Colombo M et al. Coronavirus (COVID-19) outbreak: what the department of endoscopy should know. Gastrointest Endosc 2020: doi:10.1016/j. gie.2020.03.019

[3] Christensen M, Matzen P, Schulze S et al. Complications of ERCP: a prospective study. Gastrointest Endosc 2004; 60: 721-731

[4] Rex DK, Sieber M, Lehman GA et al. A double-reprocessing high-level disinfection protocol does not eliminate positive cultures from the elevators of duodenoscopes. Endoscopy 2018; 50: 588-596

\section{Bibliography}

Endoscopy 2020; 52: 932-932

DOI 10.1055/a-1180-8681

ISSN 0013-726X

(C) 2020. Thieme. All rights reserved. Georg Thieme Verlag KG Rüdigerstraße 14, 70469 Stuttgart, Germany 